\title{
Generation of human auditory steady-state responses (SSRs). II: Addition of responses to individual stimuli
}

\author{
Rosamaria Santarelli a, Maurizio Maurizi ${ }^{\mathrm{b}}$, Guido Conti ${ }^{\mathrm{b}, *}$, Fabrizio Ottaviani ${ }^{\mathrm{b}}$, \\ Gaetano Paludetti ${ }^{b}$, Vito Enrico Pettorossi ${ }^{\mathrm{c}}$ \\ a Institute of Human Physiology, Rome, Italy

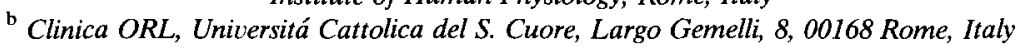 \\ ${ }^{c}$ Institute of Human Physiology, University of Perugia, Perugia, Italy
}

Received 31 March 1994; revised 5 October 1994; accepted 11 November 1994

\begin{abstract}
In order to investigate the generation of the $40 \mathrm{~Hz}$ steady-state response (SSR), auditory potentials evoked by clicks were recorded in 16 healthy subjects in two stimulating conditions. Firstly, repetition rates of 7.9 and $40 \mathrm{~Hz}$ were used to obtain individual middle latency responses (MLRs) and $40 \mathrm{~Hz}$-SSRs, respectively. In the second condition, eight click trains were presented at a $40 \mathrm{~Hz}$ repetition rate and an inter-train interval of $126 \mathrm{~ms}$. We extracted from the whole train response: (1) the response-segment taking place after the last click of the train (last click response, LCR), (2) a modified LCR (mLCR) obtained by clearing the LCR from the amplitude enhancement due to the overlapping of the responses to the clicks preceding the last within the stimulus train.

In comparison to MLRs, the most relevant feature of the evoked activity following the last click of the train (LCRs, mLCRs) was the appearance in the 50-110 ms latency range of one (in 11 subjects) or two (in 2 subjects) additional positive-negative deflections having the same periodicity as that of MLR waves.

The grand average (GA) of the $40 \mathrm{~Hz}$-SSRs was compared with three predictions synthesized by superimposing: (1) the GA of MLRs, (2) the GA of LCRs, (3) the GA of mLCRs. Both the MLR and mLCR predictions reproduced the recorded signal in amplitude while the LCR prediction amplitude resulted almost twice that of the $40 \mathrm{~Hz}-\mathrm{SSR}$. With regard to the phase, the MLR, LCR and mLCR closely predicted the recorded signal.

Our findings confirm the effectiveness of the linear addition mechanism in the generation of the $40 \mathrm{~Hz}-\mathrm{SSR}$. However the responses to individual stimuli within the $40 \mathrm{~Hz}$-SSR differ from MLRs because of additional periodic activity. These results suggest that phenomena related to the resonant frequency of the activated system may play a role in the mechanisms which interact to generate the $40 \mathrm{~Hz}-\mathrm{SSR}$.
\end{abstract}

Keywords: Middle latency responses; $40 \mathrm{~Hz}$ steady-state responses; Resonant frequency; Auditory system

\section{Introduction}

Since the first description by Galambos and coworkers (1981), the problem of the generation of the auditory $40 \mathrm{~Hz}$ Steady-State Response (SSR) has been debated without a general agreement having been reached. As previously mentioned in the first part of our study (Azzena et al., 1995), two main hypotheses have been proposed. According to the first, the 40 $\mathrm{Hz}-\mathrm{SSR}$ reflects a more general property of the sensory

\footnotetext{
${ }^{*}$ Corresponding author.
}

systems (Basar et al., 1979a; Basar et al., 1979b; Bressler and Freeman, 1980; Basar et al., 1987), behaving like neural resonators tuned to a frequency of $40 \mathrm{~Hz}$ (Galambos, 1982). However, the second hypothesis which states that the $40 \mathrm{~Hz}$ response results from the linear addition of transient middle latency responses (MLRs) elicited by individual stimuli (Galambos et al., 1981) has gained wider acceptance. This latter hypothesis is supported by the close correspondence of the SSR predicted by MLR superimposition to the recorded $40 \mathrm{~Hz}-\mathrm{SSR}$ (Galambos et al., 1981; Stapells et al., 1988; Hari et al., 1989; Ottaviani et al., 1990; Plourde et al., 1991). 
Nevertheless, as introduced in the first paper (Azzena et al., 1995), the effectiveness of predictive models based on the linear MLR addition does not rule out the possibility that phenomena related to the resonant frequency of the system may take place in the SSR generation. In other words, these two hypotheses may deal with two different features of the auditory system, and thus they do not mutually exclude each other.

Moreover, our first paper supports the hypothesis of Stapells et al. (1984), since our results highlight the role played by the relative refractory period on the SSR parameters. As a consequence, one could expect that the responses elicited by individual clicks during the SSR generation may be in some way different from the transient MLRs obtained at low repetition rates.

Thus, assuming that other mechanisms besides linear summation take place in the $40 \mathrm{~Hz}$-SSR generation, the problem becomes: What summates?

The present study was undertaken in order to isolate a basic response to individual stimuli within the 40 $\mathrm{Hz}$-SSR. To do this we performed a stimulation paradigm consisting of click trains at a $40 \mathrm{~Hz}$ rate with an inter-train interval lasting long enough to prevent the overlapping of the response following the last click of a train to those evoked by the following train. The next step was to verify the effectiveness of this isolated response in predicting the $40 \mathrm{~Hz}$-SSR by linear addition.

\section{Material and methods}

\subsection{Subjects and stimulation paradigm}

Sixteen healthy subjects ( 11 males, 5 females; aged 20-38 years) were studied. Informed consent was obtained from all subjects. None had a history of neurological or otological diseases. All had normal hearing with thresholds within $15 \mathrm{~dB} \mathrm{HL}$ at octave frequencies from 0.25 to $4 \mathrm{kHz}$ (ISO 389, 1985). The subjects were lying on a bed in a sound attenuated chamber. They were instructed to relax with their eyes closed and not to fall asleep and the state of alertness was verified throughout the recording session.

Stimuli were given monoaurally by a TDH 49 earphone. They consisted of 0.1 ms clicks with alternating polarity presented at an intensity of $105 \mathrm{~dB}$ p.e. SPL (sound level meter Brüel and Kjaer 2231, artificial ear Brüel and Kjaer 4152) corresponding to $80 \mathrm{~dB} \mathrm{nHL}$ in reference to the psychoacoustical threshold of normal hearing subjects.

In the first part of the recording session stimuli were presented at repetition rates of 7.9 and $40 \mathrm{~Hz}$ in order to obtain respectively basic MLRs and $40 \mathrm{~Hz}$-SSRs.

In the second part of the recording session stimuli consisted of trains of eight clicks with a $40 \mathrm{~Hz}$ repetition rate and each train was presented every $301 \mathrm{~ms}$; as a consequence the time interval between the last click of each train and the first click of the following one (inter-train interval) lasted as long as $126 \mathrm{~ms}$ (i.e., the same interval utilized to obtain MLRs at $7.9 \mathrm{~Hz}$ of repetition rate).

\subsection{Recording}

Electroencephalographic activity was recorded ipsilaterally to the stimulated ear using silver-chloride cup electrodes attached to the scalp with saline gel. The active electrode was applied to the vertex $(\mathrm{Cz})$, the reference electrode was placed on the right or left mastoids (M1 or M2) and the ground electrode was placed on the forehead. Inter-electrode impedance was kept below 5 kOhms.

Signals were amplified (100 000) and filtered (5-3000 $\mathrm{Hz}, 12 \mathrm{~dB}$ /octave). Analogue to digital conversion was performed at a sample rate of $5 \mathrm{kHz}$ (evoked potentials recording equipment EAC Mercury MDP 11/23 plus). Artefact rejection was active in order to avoid recording of movement artefacts.

Two thousands epochs were averaged to obtain MLRs and $40 \mathrm{~Hz}$-SSRs. At $7.9 \mathrm{~Hz}$ each waveform was replicated once in all the tested subjects. The time analysis window was kept at $125 \mathrm{~ms}$.

When recording the responses to train stimuli, 4 blocks, each consisting of 500 epochs, were averaged. This was done in order to roughly test the waveform replicability, thus preventing the subjects from undergoing a long lasting recording session. The time analysis window was $300 \mathrm{~ms}$.

\subsection{Data analysis}

Individual MLRs were obtained by averaging the two replicated waveforms. The MLR components were labelled according to their polarity and in alphabetical order (Mendel and Goldstein, 1971; Thornton et al., 1977) even if the latency of the components may make them seem to belong to the slow potentials category (Picton et al., 1974; Picton and Smith, 1978). Wave V of the auditory brainstem response (ABR) and the $P$ wave preceding $\mathrm{Na}$ (Picton et al., 1974; Picton and Smith, 1978; Kavanagh et al., 1984) were also identified.

Individual responses to stimulus trains were obtained by averaging the 4 blocks recorded in each subject. The $125 \mathrm{~ms}$ time window (625 point-segment) following the last click of the stimulus train was extracted from the whole train response by cutting the first 875 point-segment corresponding to the first 175 $\mathrm{ms}$ of the record-length (Fig. 1). The resulting curve was arbitrarily indicated as last click response (LCR) 

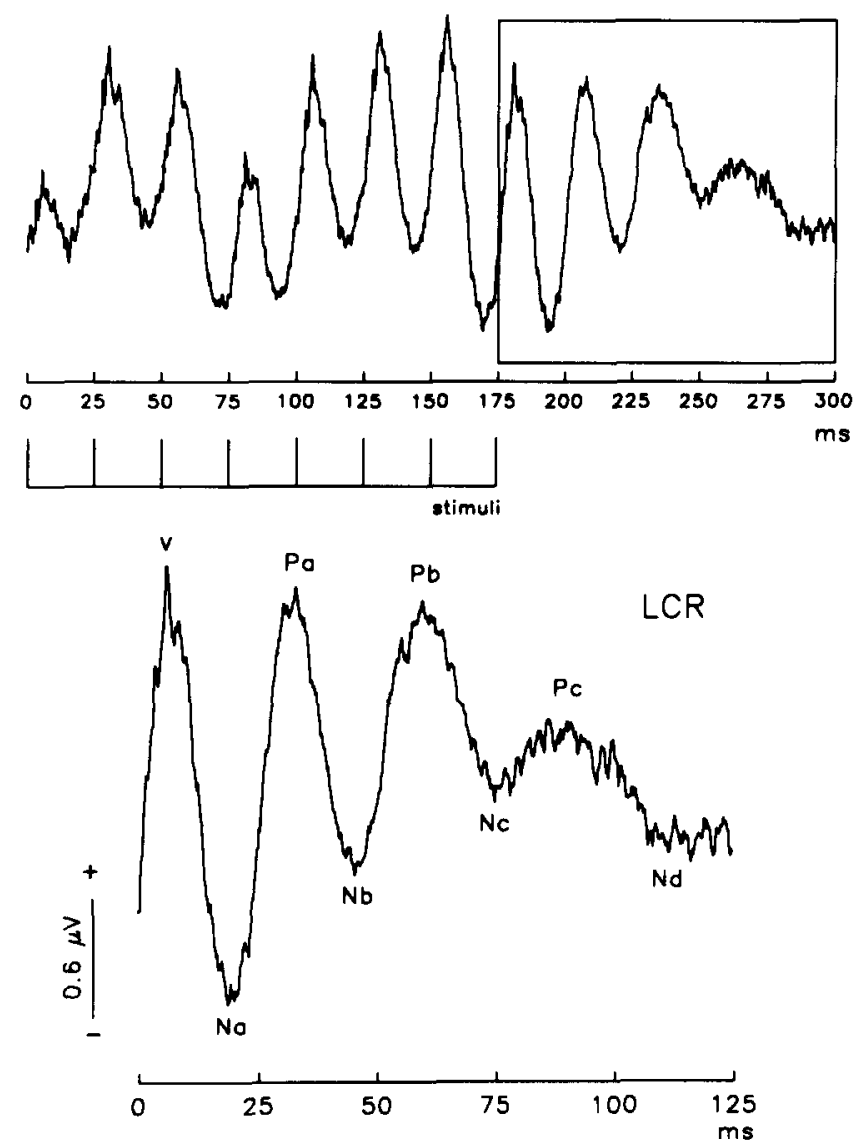

Fig. 1. Representative last click response from one subject (Subj. 11). In the upper part of the figure the whole response to the eight-click train is reported. The square encloses the $125 \mathrm{~ms}$ time window following the last click of the train. The recorded response is shown magnified in the lower part of the figure. The calibration bar refers to the magnified trace. Upward deflection indicates vertex positivity in this figure and in the subsequent ones.

and its components were labelled as the corresponding MLR waves observed in the same latency range.

Latency and amplitude values of MLR and LCR components were calculated in each subject. The amplitude was measured from one peak to the following one of opposite polarity except the first positive-negative deflection. In this case we measured the amplitude from wave $\mathrm{V}$ to the following $\mathrm{Na}$ due to the high variability in $P$ wave detectability and amplitude. $T$ paired test was performed in order to compare latency and amplitude values of MLR and LCR components. Differences were considered significant at $P<0.01$.

In each subject, prediction curves of the $40 \mathrm{~Hz}$-response were obtained by the linear addition of MLR and LCR traces, respectively. The 625 point-MLR (and LCR) curve was divided into five consecutive 125 point-segments, each corresponding to $25 \mathrm{~ms}$. Then five consecutive 625 point-curves were summed in such a way that each curve was shifted by $25 \mathrm{~ms}$ with respect to the previous one (see Fig. 1 in Azzena et al., 1995).
The Fourier series technique was applied to the recorded and synthesized curves to evaluate amplitude and phase values of the fundamental frequency (see paper one for details). Amplitude and phase values were expressed in $\mu \mathrm{V}$ and radians, respectively.

\section{Results}

3.1. Middle latency responses (MLRs) and last click responses (LCRs): main features, latency and amplitude values

The whole response to $40 \mathrm{~Hz}$-trains obtained in two representative subjects together with the grand average (GA) of all individual train responses are illustrated in Fig. 2. One can observe that the activity within the first $25 \mathrm{~ms}$ is mainly represented by the ABR. The addition of the responses to consecutive stimuli takes place after the second stimulus so that a good synchronization is obtained within the first $100 \mathrm{~ms}$. These observa-
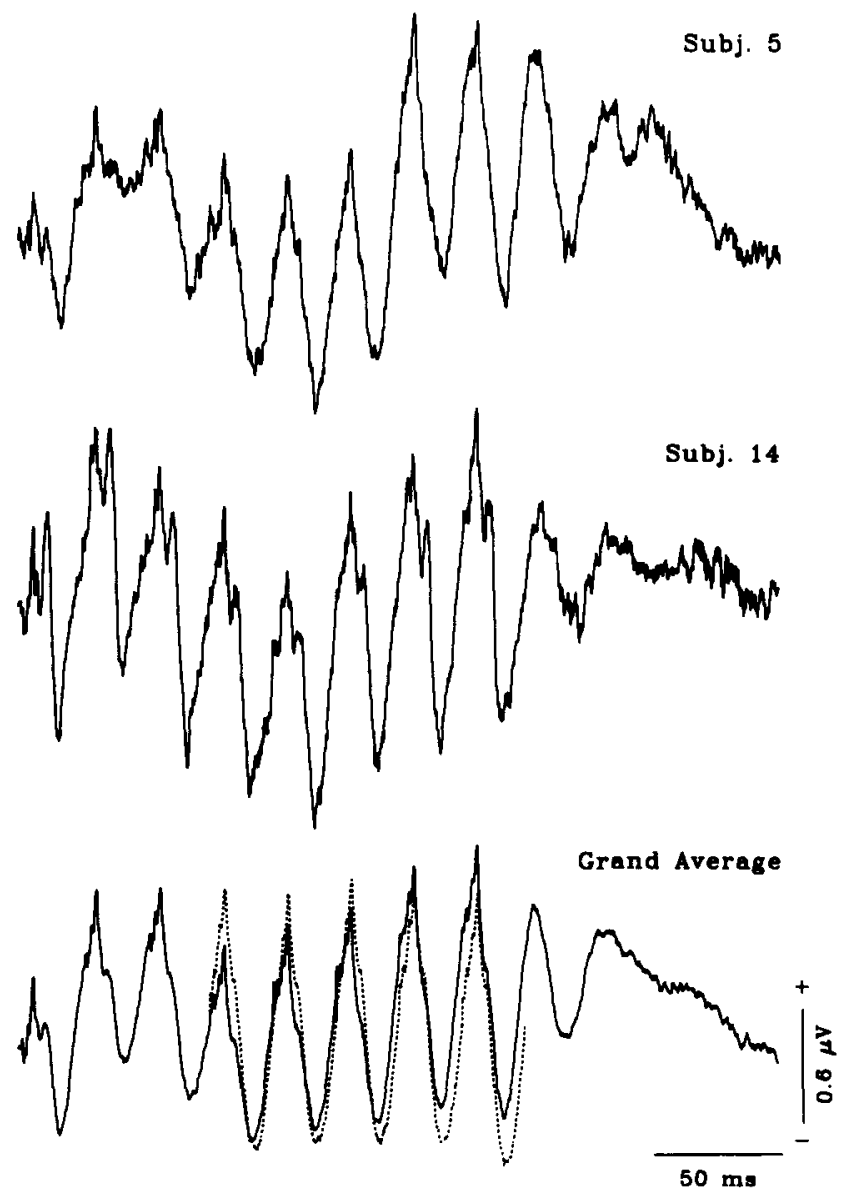

Fig. 2. Whole responses to the eight-click train. Grand average from all subjects and representative individual responses from two subjects are shown. Grand average of the $40 \mathrm{~Hz}-\mathrm{SSRs}$ (dashed line) is superimposed to the train response in the $75-200 \mathrm{~ms}$ time interval. 
tions are in agreement with the findings obtained by Mäkelä and Hari in cats (1987), and by Mäkelä et al. in humans (1990) when utilizing a stimulus train paradigm. As is shown in Fig. 2, the GA of the $40 \mathrm{~Hz}$-SSRs closely resembles the segment of the GA of the train responses corresponding to the last clicks of the train. In particular, no significant amplitude or phase differences between the $40 \mathrm{~Hz}$-SSR and the last two periods (150-200 ms time interval) within the train were found. The periodic $40 \mathrm{~Hz}$ activity within the train response appears to be superimposed on an alternating positive (at about $50 \mathrm{~ms}$ )-negative (at about $100 \mathrm{~ms}$ )-positive (at about $200 \mathrm{~ms}$ ) slow response (Fig. 2). Taking into account our filter-setting $(5-3000 \mathrm{~Hz}$ pass-band), this activity may be interpreted as corresponding to the slow responses (P40, N100 and P200) isolated by Mäkelä and Hari (1987) by filtering the whole train response with a $0.05-100 \mathrm{~Hz}$ pass-band. A further analysis of this slow activity was not carried out since it was beyond the aims of our study.

Representative MLRs and LCRs from 4 subjects are illustrated in Fig. 3. LCRs differed from the corre-

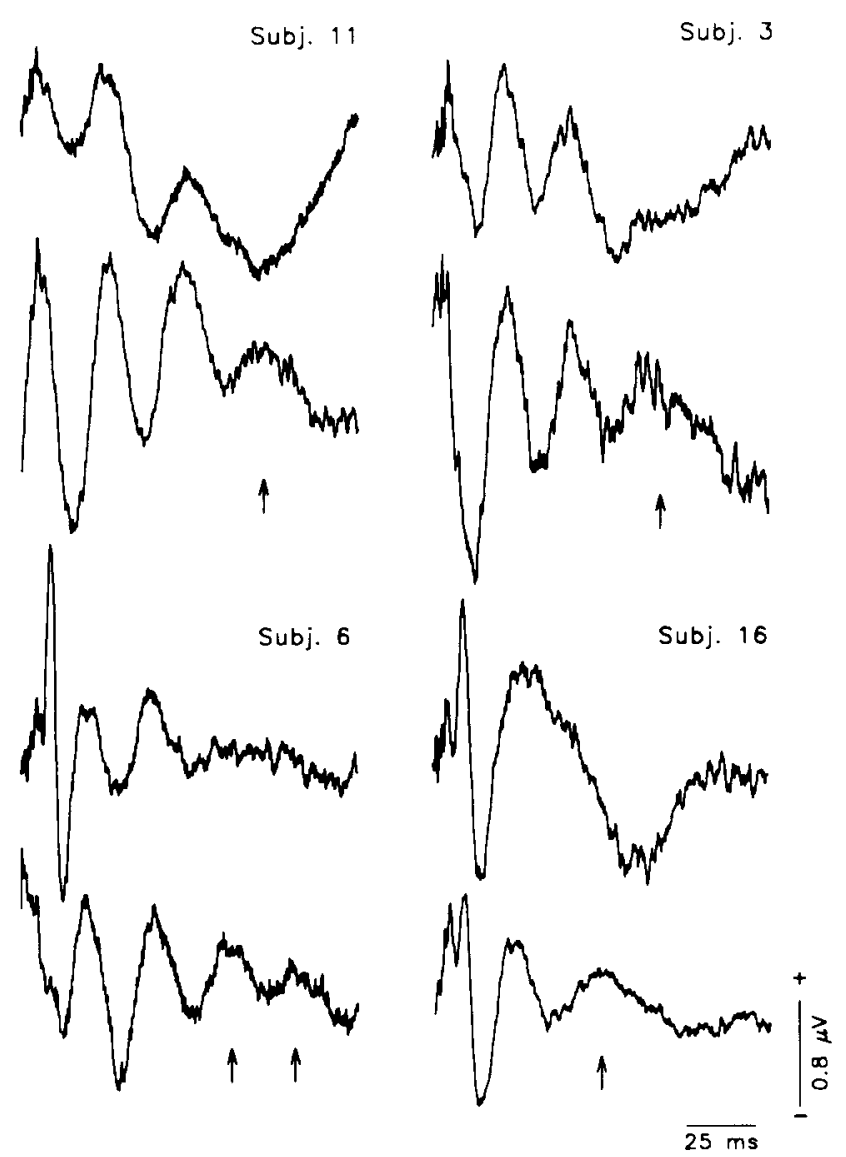

Fig. 3. Middle latency responses (MLRs) and last click responses (LCRs). MLRs (upper trace) and LCRs (lower trace) from 4 representative subjects are shown. Arrows indicate the additional positive components seen in LCRs in comparison to MLRs.
Table 1

Identification of middle latency response (MLR) and last click response (LCR) main components

\begin{tabular}{|c|c|c|c|c|c|}
\hline & \multicolumn{2}{|c|}{ MLRc } & \multicolumn{3}{|c|}{ LCRs } \\
\hline & $\mathrm{Pb}$ & $\overline{P c}$ & $\overline{\mathrm{Pb}}$ & $\mathrm{Pc}$ & $P d$ \\
\hline Subj & + & + & + & + & - \\
\hline 1 & + & + & + & + & - \\
\hline 2 & + & + & + & + & - \\
\hline 3 & + & - & + & + & - \\
\hline 4 & + & - & + & + & - \\
\hline 5 & + & - & + & + & - \\
\hline 6 & + & - & + & + & + \\
\hline 7 & + & + & + & + & + \\
\hline 8 & + & + & + & + & - \\
\hline 9 & + & - & + & + & - \\
\hline 10 & + & - & + & + & - \\
\hline 11 & + & - & + & + & - \\
\hline 12 & + & - & + & + & - \\
\hline 13 & - & + & + & + & - \\
\hline 14 & - & - & + & + & - \\
\hline 15 & - & - & + & - & - \\
\hline 16 & - & - & + & - & - \\
\hline
\end{tabular}

Presence $(+)$ or absence $(-)$ of the main positive components in MLR and LCR are compared for each subject. Pa wave was not reported as it was identified in both MLRs and LCRs in all subjects.

sponding MLRs in two main aspects. First, LCRs showed higher amplitude of the segment $\mathrm{Pa}-\mathrm{Nb}$ and, above all, of the segment V-Na. However, the most relevant feature of LCRs was the appearance of one or two deflections in addition to those already present in the corresponding MLRs (Table 1). In fact, besides the Pa wave which was always identifiable in both MLRs and LCRs, the observations increased from 12 to 16 for the $\mathrm{Pb}$ wave and from 5 to 14 for the $\mathrm{Pc}$ wave when comparing MLRs to LCRs. The most commonly observed pattern in LCRs ( 9 subjects) was the appearance in the $\mathrm{Pc}$ latency range of an additional wave which was absent in the corresponding MLRs. This is clearly seen for subjects 3 and 11 (Fig. 3).

The LCR did not show a Pc component in two subjects. However a $\mathrm{Pb}$ wave which was not identifiable in the corresponding MLRs was found (see, for example subject 16 in Fig. 3). In one subject, only the $\mathrm{Pa}$ component was identifiable in the MLR while the LCR showed additional $\mathrm{Pb}$ and $\mathrm{Pc}$ waves. Moreover, in the LCRs of two subjects, further deflections in addition to Pc (and Nd) were found. These components were indicated as $\mathrm{Pd}$ and $\mathrm{Ne}$ according to alphabetical order (see for example subject 6 in Fig. 3).

It should be remarked that all the additional components showed a good replicability as they were easily identifiable in all the four blocks recorded in each subject.

In conclusion, in 13 out of 16 subjects, LCRs showed one (11 subjects) or two ( 2 subjects) additional components in comparison to the corresponding MLRs.

Mean and standard deviations of amplitude and 
Table 2

Latency values of middle latency response (MLR), last click response (LCR) and modified last click response (mLCR) components

\begin{tabular}{|c|c|c|c|c|c|c|c|c|c|c|}
\hline \multicolumn{11}{|c|}{ Latency (ms) } \\
\hline MLRs & V & $\mathrm{Na}$ & $\mathrm{Pa}$ & $\mathrm{Nb}$ & $\mathrm{Pb}$ & $\mathrm{Nc}$ & $\mathrm{Pc}$ & $\mathrm{Nd}$ & $\mathrm{Pd}$ & $\mathrm{Ne}$ \\
\hline$N$ & 16 & 16 & 16 & 16 & 12 & 12 & 5 & 5 & - & - \\
\hline Mean & 5.92 & 17.36 & 28.61 & 45.29 & 51.00 & 68.51 & 90.09 & 112.29 & - & - \\
\hline STD & 0.25 & 1.21 & 2.77 & 11.44 & 4.75 & 10.63 & 8.01 & 5.11 & - & - \\
\hline \multicolumn{11}{|l|}{ LCRs } \\
\hline$N$ & 16 & 16 & 16 & 16 & 16 & 16 & 14 & 14 & 2 & 2 \\
\hline Mean & 6.00 & 17.05 & 28.77 & 41.71 & 57.38 & 73.67 & 85.41 & 107.39 & 104.25 & 120.06 \\
\hline STD & 0.28 & 1.70 & 1.98 & 3.46 & 4.48 & 9.06 & 10.06 & 12.64 & 4.60 & 2.46 \\
\hline \multicolumn{11}{|c|}{ mLCRs } \\
\hline$N$ & 16 & 16 & 16 & 16 & 16 & 16 & 14 & 14 & 2 & 2 \\
\hline Mean & 6.06 & 17.94 & 27.76 & 41.31 & 58.63 & 74.95 & 90.17 & 111.70 & 104.55 & 2.66 \\
\hline STD & 0.25 & 2.05 & 1.73 & 3.26 & 5.85 & 9.21 & 8.52 & 10.52 & 3.53 & 2.66 \\
\hline
\end{tabular}

Means, Standard deviations (STD) and the total number of observations $(N)$ are reported for each component.

latency values of MLR components are reported in Tables 2 and 3, respectively. Wave $V$ of $A B R$ was always easily identifiable within the first $15 \mathrm{~ms}$ of the response (mean latency $5.92 \pm 0.25 \mathrm{~ms}$; mean amplitude $0.27 \pm 0.10 \mu \mathrm{V})$. The $\mathrm{P}$ wave preceding the $\mathrm{Na}$ peak (Kavanagh et al., 1984) was identified in 12 subjects (latency $11.52 \pm 1.32 \mathrm{~ms}$; amplitude $0.94 \pm 0.75$ $\mu \mathrm{V})$. Because of its high voltage and amplitude variability, this $\mathbf{P}$ wave may be considered myogenic in seven subjects.

Mean and standard deviations of amplitude and latency values of LCR components are reported in Tables 2 and 3, respectively. No significant differences in peak latency values were found between MLRs and LCRs except for the $\mathrm{Pb}$ wave which was delayed in LCRs in comparison to MLRs $(P<0.01)$. With regards to amplitudes, LCRs gave higher values for V-Na, $\mathrm{Na}-\mathrm{Pa}, \mathrm{Pa}-\mathrm{Nb}$ and $\mathrm{Nb}-\mathrm{Pb}$ compared to MLRs. The difference was statistically significant for V-Na $(P<$ $0.001)$, Na-Pa $(P<0.01)$ and $\mathrm{Nb}-\mathrm{Pb}(P<0.0001)$.

The amplitude differences observed between MLRs and LCRs may be justified assuming that the responses to individual stimuli summate linearly within LCRs. In this case the LCR would result from the overlapping of the responses elicited by the last clicks of the train. To obtain a curve which could be considered an approximation to the response evoked by individual clicks within the SSR, an analytical handling was performed on the LCR trace. Thus a modified LCR (mLCR) was obtained by subtracting the LCR curve taken without delay from the same curve shifted by $25 \mathrm{~ms}$, as indicated in Fig. 4.

Representative mLCRs together with the corresponding MLRs from 4 subjects are illustrated in Fig. 5. In comparison with LCRs, mLCRs showed a more strict resemblance to MLR morphology due to a decrease in amplitude of its early components. However, as was expected, mLCRs differed from MLRs since they showed the same additional components found in LCRs in each subject. Mean and standard deviations of amplitude and latency values of mLCR components are reported in Tables 2 and 3, respectively. The mLCR components did not show significant differences in latency in comparison to LCR and MLR waves except

Table 3

Amplitude values of middle latency response (MLR), last click response (LCR) and modified last click response (mLCR) components

\begin{tabular}{|c|c|c|c|c|c|c|c|c|c|}
\hline \multicolumn{10}{|c|}{ Amplitude $(\mu \mathrm{V})$} \\
\hline MLRs & $\mathrm{V}-\mathrm{Na}$ & $\mathrm{Na}-\mathrm{Pa}$ & $\mathrm{Pa}-\mathrm{Nb}$ & $\mathrm{Nb}-\mathrm{Pb}$ & $\mathrm{Pb}-\mathrm{Nc}$ & $\mathrm{Nc}-\mathrm{Pc}$ & Pc-Nd & $\mathrm{Nd}-\mathrm{Pd}$ & $\mathrm{Pd}-\mathrm{Ne}$ \\
\hline$N$ & 16 & 16 & 16 & 12 & 12 & 5 & 5 & - & - \\
\hline Mean & 0.92 & 0.92 & 0.89 & 0.41 & 0.60 & 0.57 & 0.57 & - & - \\
\hline STD & 0.34 & 0.44 & 0.46 & 0.25 & 0.25 & 0.31 & 0.33 & - & - \\
\hline \multicolumn{10}{|l|}{ LCRs } \\
\hline$N$ & 16 & 16 & 16 & 16 & 16 & 14 & 14 & 2 & 2 \\
\hline Mean & 1.57 & 1.29 & 0.97 & 0.87 & 0.54 & 0.34 & 0.58 & 0.14 & 0.36 \\
\hline STD & 0.56 & 0.42 & 0.27 & 0.33 & 0.27 & 0.19 & 0.26 & 0.08 & 0.09 \\
\hline \multicolumn{10}{|c|}{ mLCRs } \\
\hline$N$ & 16 & 16 & 16 & 16 & 16 & 14 & 14 & 2 & 2 \\
\hline Mean & 0.99 & 0.72 & 0.91 & 1.08 & 0.59 & 0.69 & 0.53 & 0.33 & 0.53 \\
\hline STD & 0.54 & 0.34 & 0.31 & 0.42 & 0.31 & 0.27 & 0.15 & 0.05 & 0.13 \\
\hline
\end{tabular}

Means, Standard deviations (STD) and the total number of observations $(N)$ are reported for each component. 
$\mathrm{Pb}$ which was significantly delayed in mLCRs in comparison to MLRs. With respect to amplitude, mLCRs showed significantly lower values for $\mathrm{V}-\mathrm{Na}(P<0.0001)$ and $\mathrm{Na}-\mathrm{Pa}(P<0.0001)$ and higher values for $\mathrm{Nc}-\mathrm{Pc}$ $(P<0.0001)$ in comparison to mLCRs. When compared with MLRs, LCRs showed significantly higher values only for $\mathrm{Nb}-\mathrm{Pb}(P<0.001)$.

\section{2. $40 \mathrm{~Hz}$-SSR predictions}

Three predictions of the $40 \mathrm{~Hz}-\mathrm{SSR}$ were calculated utilizing GA of MLRs, LCRs and mLCRs, respectively, as the basic response (Fig. 6).

Prediction curves and GA of the recorded $40 \mathrm{~Hz}$ SSRs are shown on the right side of Fig. 6 . Amplitude and phase values were calculated on the fundamental frequency of the $40 \mathrm{~Hz}$-SSR (amplitude $0.61 \mu \mathrm{V}$; phase $4.502 \mathrm{rad}$ ) and of each synthetic curve. Results showed that the MLR prediction reproduced the recorded response in amplitude $(0.54 \mu \mathrm{V})$ and phase $(4.381 \mathrm{rad})$. LCR prediction showed a phase value $(4.276 \mathrm{rad})$ very close to that of the measured $40 \mathrm{~Hz}-\mathrm{SSR}$; amplitude
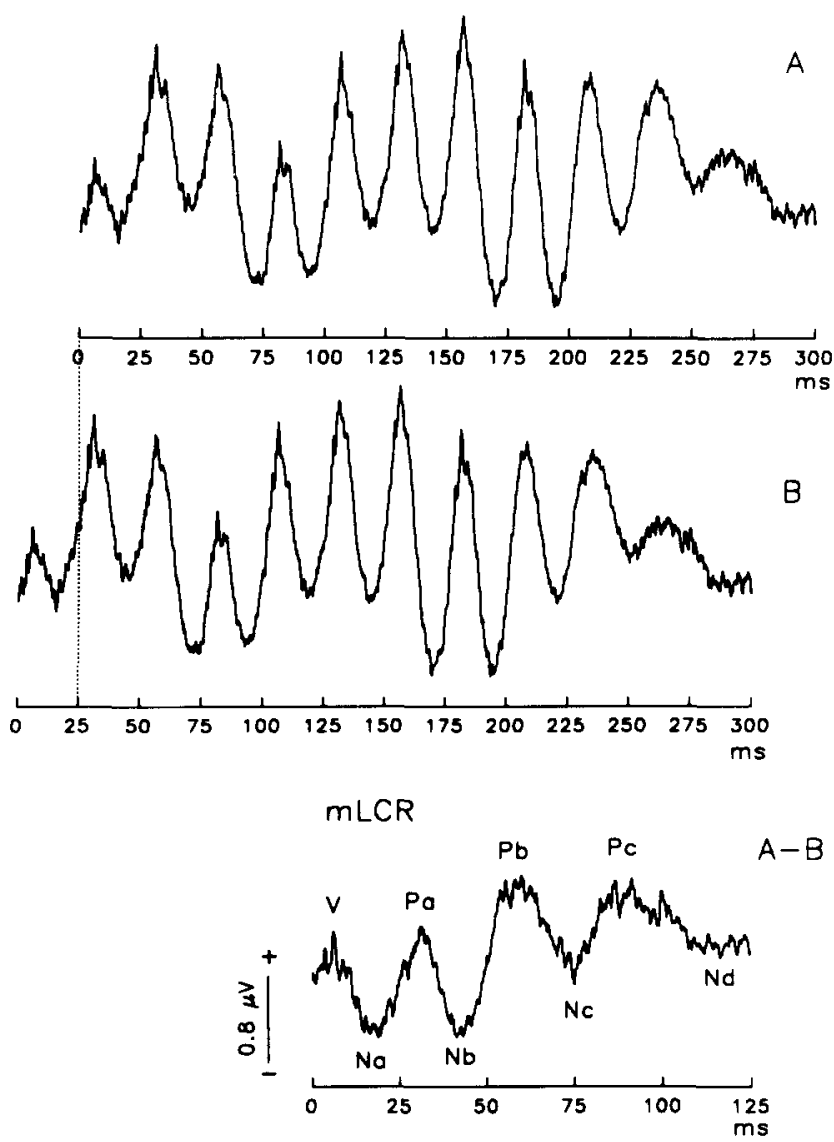

Fig. 4. Representative modified last click response (mLCR) from one subject (Subj. 11). The whole response to train stimulation $(300 \mathrm{~ms}$ time-window; 1500 point-trace) (B) was subtracted from the same response shifted by $25 \mathrm{~ms}$ (125 point-segment) (A). The last $125 \mathrm{~ms}$ of the resulting curve ( 625 point-segment) (A-B) represents mLCR.
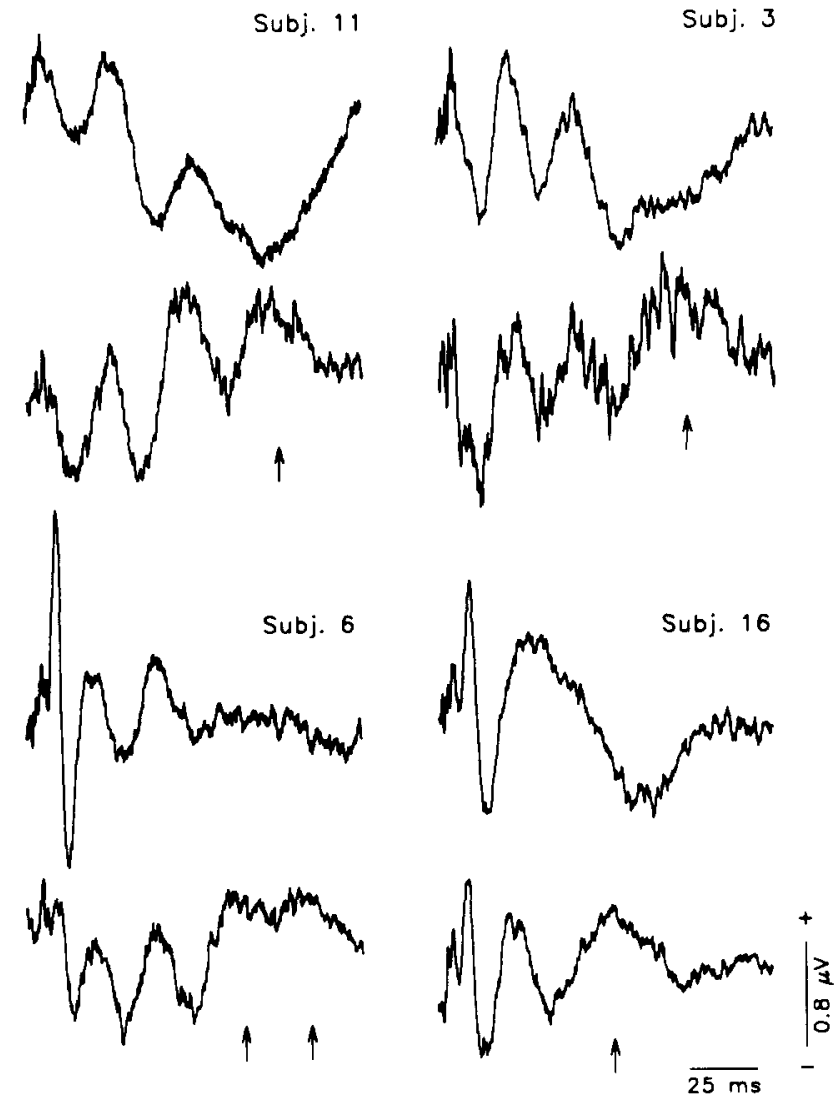

Fig. 5. Middle latency responses (MLRs) and modified last click responses (mLCRs). MLR (upper trace) and mLCR (lower trace) from 4 representative subjects (the same as in Fig. 3) are shown. Arrows indicate the additional positive components identified in mLCR in comparison to MLRs.

(1.11 $\mu \mathrm{V})$ instead resulted almost twice that of the recorded response. This amplitude difference disappeared when the prediction was synthesized utilizing GA of mLCRs as the basic response. In fact, the mLCR prediction curve reproduced the recorded 40 $\mathrm{Hz}-\mathrm{SSR}$ in both amplitude $(0.61 \mu \mathrm{V})$ and phase $(4.276$ $\mathrm{rad})$.

\section{Discussion}

Our study was aimed at isolating the 'basic response' to individual stimuli within the $40 \mathrm{~Hz}$-SSR and at verifying if some differences exist between this 'basic response' and the transient MLR evoked at lower repetition rates. To this aim, a paradigm based on a train stimulation offers a suitable model since it is characterized by an abrupt interruption of the stimuli sequence after reaching the steady-state. This condition appears to be achieved in our paradigm since the last periods of the train strictly resembled the $40 \mathrm{~Hz}$ SSR in morphology as well as in amplitude and phase. As a consequence, the response-segment following the 


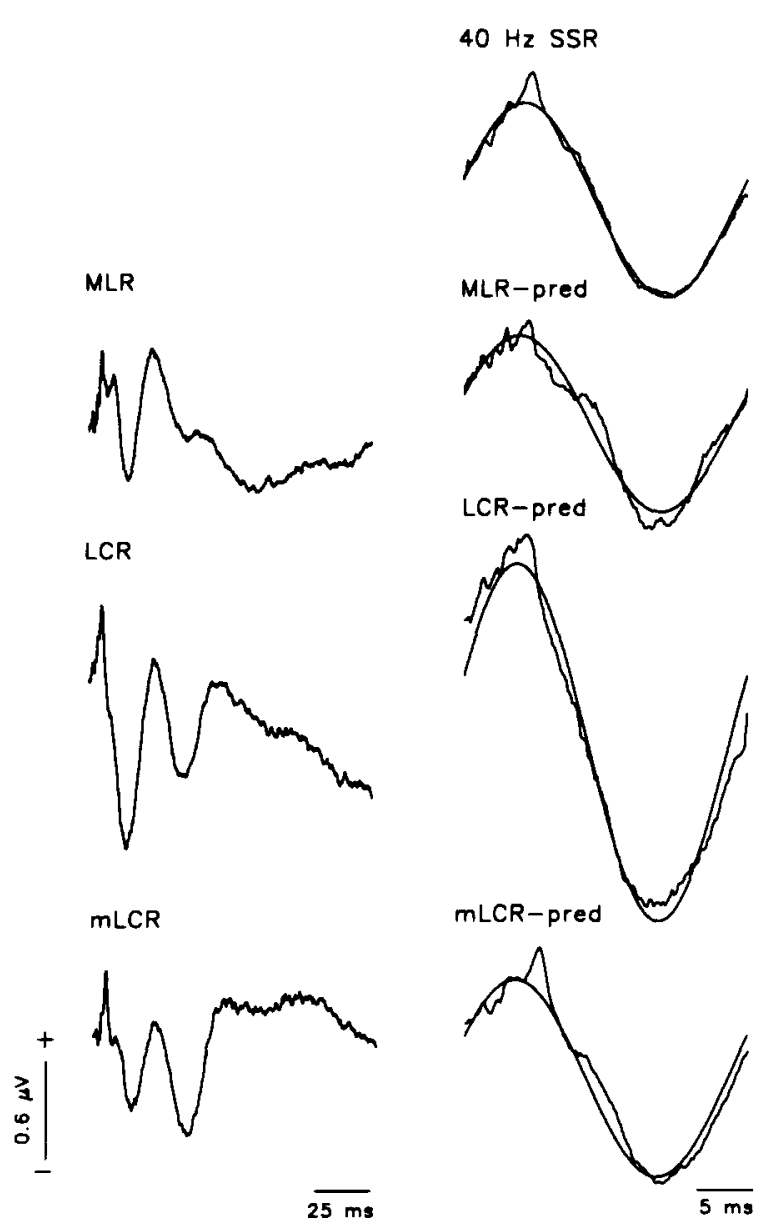

Fig. 6. Grand average of the recorded $40 \mathrm{~Hz}$-SSR and predicted curves obtained from middle latency responses (MLRs), last click responses (LCRs) and modified last click responses (mLCRs) are shown on the right side from the top to the bottom. On the left side Grand averages of the basic responses (MLRs, LCRs and mLCRs respectively) from which the predicted curves were obtained are illustrated. For the recorded and predicted $40 \mathrm{~Hz}$-SSR only one cycle is reported and the first harmonic curve is superimposed. Amplitude calibration bar refers to all the reported traces.

last stimulus of the train reflects the properties of the responses to individual stimuli when the system is in the steady-state condition.

\subsection{Comparison between MLRs, LCRs and $m L C R s$}

The analysis of the responses following the last click of the train allows us to identify several differences with respect to MLRs. The most relevant was found in the late portion of the response where LCRs showed one or two additional components with respect to MLRs in the $50-110 \mathrm{~ms}$ latency range in 13 out of 16 subjects. LCRs behaved in the following way in 9 subjects: an additional component which was in the same latency range of the Pc wave of MLRs followed the $\mathrm{Pb}$ wave. This additional activity seemed to maintain the same periodicity as that of MLRs since the inter-peak latencies $\mathrm{Pb}-\mathrm{Pc}$ and $\mathrm{Pc}-\mathrm{Pd}$ were similar, ranging from 20 to $30 \mathrm{~ms}$. Interestingly, in two cases LCRs showed an additional positive component following the Pc wave. This component which was labelled Pd, has not been previously described in MLR studies.

The reported morphological differences suggest that MLRs and LCRs may represent the output of the same system under two states of activation. As a consequence, the comparison between MLRs and LCRs with regard to latency and amplitude values deserves caution. Nevertheless, with respect to latency, the only significant difference consisted in a delayed $\mathrm{Pb}$ component in LCRs. In our previous study, phenomena dealing with the recovery cycle have been shown to take place to determine amplitude and latency of the steady-state auditory responses (Azzena et al., 1995). Taking into account these data, one can hypothesize that the increased $\mathrm{Pb}$ latency may result from a rate effect taking place at $40 \mathrm{~Hz}$. Moreover, with regard to amplitude, it should be noted that LCR components show an increase in comparison to the corresponding MLR waves within the first $50 \mathrm{~ms}$ and this difference attains the significance for $\mathrm{V}-\mathrm{Na}$ and $\mathrm{Nb}-\mathrm{Pb}$. This was explained taking into account that the $\mathrm{LCR}$ results from the superimposition of the responses elicited by the last clicks of the train. On the basis of this assumption and admitting the attainment of the steady state, the analytical handling performed on LCR allowed us to isolate the response elicited by the last click of the train. Thus the extracted mLCR would represent the basic response to individual stimuli within the $40 \mathrm{~Hz}$ SSR. In fact, in the first $50 \mathrm{~ms}$ mLCRs showed several waves which were comparable in latency and amplitude values to ABR (wave V) and MLR waves. Nevertheless mLCRs still differ from MLRs because of the additional components found in the late part of the responses and the increased $\mathrm{Nb}-\mathrm{Pb}$ amplitude.

Therefore it could be suggested that the main difference between the responses elicited by individual stimuli at low rates (MLRs) and those obtained at high rates (mLCRs) consists of the appearance of additional neural activity during steady-state stimulation. Additional activity following steady-state responses has not been described in previous studies utilizing a stimulus train paradigm in cats (Mäkelä et al., 1990) and in humans (Mäkelä and Hari, 1987).

Given the absolute latency of the additional components present in LCRs and in mLCRs, one of the most important questions deals with the possibility that these components correspond to slow potentials elicited by the first click of the train. However this hypothesis seems unlikely. It is reasonable to assume that the recovery cycle of the response to the first click of the train is determined, at the most, by the inter-train interval. This, in fact, lasts as long as the inter-stimulus interval utilized in MLR recordings. Thus, if a P2 
component is elicited during train stimulation, one would also find it in MLRs. This view is supported by previous studies which have reported that a $\mathrm{P} 2$ wave does not become detectable until an inter-stimulus interval of about $200 \mathrm{~ms}$ (Picton et al., 1992), which is longer than that utilized in our experiment. Neverthless, in order to test the hypothesis that slow components may be involved with the appearance of additional activity found in LCR, a supplementary recording session was carried out based on the assumption that the recovery cycle is regulated by the repetition rate of the train stimuli. We recorded the responses to clicks presented with an inter-stimulus interval of 301 $\mathrm{ms}$ on a $300 \mathrm{~ms}$ time base in three of the previously tested subjects (Subjs. 5, 10, 11). We also recorded the MLRs and the responses to train stimulation utilizing the same paradigm described above. Both MLRs and LCRs replicated the behaviour observed in the first recording session. With regard to the transient responses obtained with an inter-stimulus interval of 301 ms, a clear $N_{1}-P_{2}-N_{2}$ complex was always detectable in

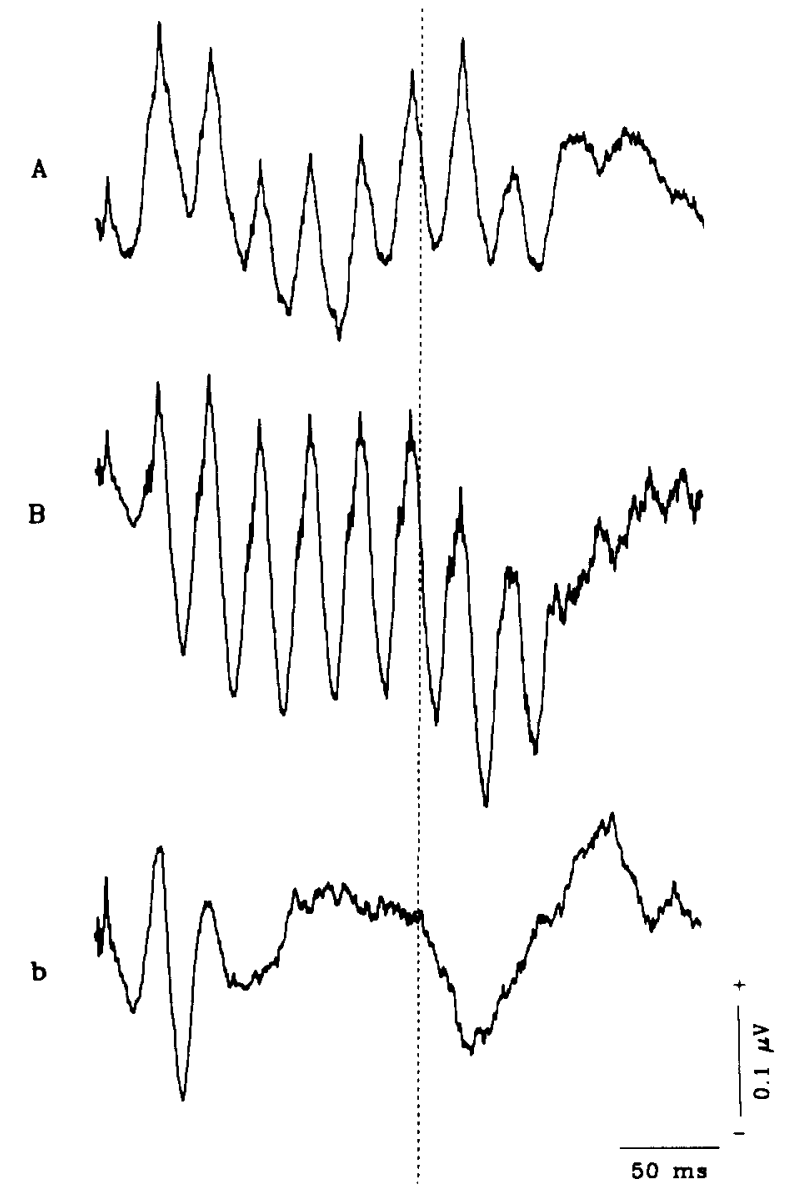

Fig. 7. Whole response to click train (A) and synthesized train response (B) from a representative subject (Subj. 10). B was obtained from a transient response elicited by clicks presented with an interstimulus interval of $301 \mathrm{~ms}$ (b) (see text for explanation). the 70-200 ms latency range. A prediction of the response to train stimulation was then synthesized by adding eight transient responses recorded at an interstimulus interval of $301 \mathrm{~ms}$, each shifted by $25 \mathrm{~ms}$ with respect to the previous one. Synthetic train responses always attained a good synchronization within the first $50 \mathrm{~ms}$. This is shown for a representative subject in Fig. 7. However, it is relevant that no additional components with respect to MLR waves were found in the latency range corresponding to LCR. This result further suggests that a residual slow cortical activity evoked by the clicks which precede the last cannot account for additional components found in LCRs.

The latency range of the observed additional activity raises the question of its relationship with the transient gamma band response (GBR) which has been recently discovered by electric (Makeig and Galambos, 1989) and magnetic (Pantev et al., 1991) encephalographic studies. It has been suggested that this response does not participate in the generation of the $40 \mathrm{~Hz}-\mathrm{SSR}$ since these neural activities originate from separate cortical sources (Pantev et al., 1993). Moreover, the GBR has been proven to be strongly enhanced by stimulus rates lower than $1 \mathrm{~Hz}$ (Makeig and Galambos, 1989; Pantev et al., 1993) and this makes it unlikely that this response appears at the rates used in our stimulation paradigm.

Another point deserving some comment concerns the state of alertness of our tested subjects. In fact, it has been reported that variations in amplitude of MLR components may be dependent on the state of wakefulness (Erwin and Buchwald, 1986). This effect cannot justify the observed differences between MLRs and LCRs as all our subjects remained awake during the whole recording session.

Thus the additional components found in LCRs and mLCRs may be regarded as the activity of a neural generator whose output is related to the stimulation period. This suggests that mechanisms related to resonant properties of the auditory system may come into play, enhancing the contribution of individual responses within the $40 \mathrm{~Hz}-\mathrm{SSR}$. Assuming linearity and admitting that the system behaves as a damped harmonic oscillator having resonant frequency of $40 \mathrm{~Hz}$ and damping ratio between 0 and 1 , one can speculate that, for a given damping value, the output modulus depends on the frequency components of the input as well as on the system resonant frequency. The increase in the input of the frequency components close to the system resonant frequency causes the output modulus to increase and this makes the appearance of further oscillations in the system output more likely.

\section{2. $40 \mathrm{~Hz}-S S R$ predictions}

The hypothesis of linear addition of individual MLRs is the most widely accepted to explain the generation 
of $40 \mathrm{~Hz}-\mathrm{SSR}$ (Galambos et al., 1981; Stapells et al., 1988; Hari et al., 1989; Plourde et al., 1991). Our results do not reject this hypothesis since the synthetic $40 \mathrm{~Hz}$-SSR obtained by the linear addition of MLR traces adequately predicted amplitude and phase of the recorded SSR. However, in our study, the initial question was: what summates to generate the $40 \mathrm{~Hz}$ SSR? To answer this question we utilized the stimulation train paradigm to isolate a basic response after the system has attained the steady state. LCR closely predicted in phase the actual $40 \mathrm{~Hz}-\mathrm{SSR}$ while its amplitude resulted almost twice that of the recorded response. However, LCRs may result from the superimposition of the neural activity elicited by the clicks preceding the last one. As a matter of fact, when utilizing the extracted $\mathrm{mLCR}$ to synthesize the 40 $\mathrm{Hz}-\mathrm{SSR}$, a close correspondence between the recorded and predicted amplitude and phase was found. Hence one can suggest that the basic responses which summate to generate the $40 \mathrm{~Hz}-S S R s$ should be identified in mLCRs rather than in MLRs. As discussed above, the mLCR greatly differs from MLR due to the presence of additional components which may be regarded as the result of an enhancing effect related to the resonant properties of the generating system.

Moreover, our previous paper suggests that adaptation phenomena take place to determine the SSR parameters starting from stimulus rates lower than 40 $\mathrm{Hz}$ (Azzena et al., 1995). One would expect that these phenomena would lead to an amplitude decrease of the actual $40 \mathrm{~Hz}-\mathrm{SSR}$ in comparison to the $40 \mathrm{~Hz}-\mathrm{SSR}$ predicted on the basis of the transient MLRs. However this was not the case. Hence it is possible to admit that the inhibitory action caused by adaptation is counterbalanced by the facilitating effect due to the resonant properties of the system.

\section{Conclusion}

The linear addition of the responses elicited by individual stimuli can still be considered to be the main mechanism underlying the SSR generation. However, other phenomena are involved so that the responses to individual stimuli within the SSRs differ from the responses recorded at rates which prevent their overlapping (MLRs). First of all, mechanisms related to the resonant frequency of the auditory system come into play, enhancing the contribution of individual responses. Furthermore, taking into account the results of our first paper, the SSR generation appears to be even more complex since nonlinearities related to the recovery cycle can determine parameters of individual responses within the steady-state potentials.

In conclusion, the surface-recorded SSRs result from the linear summation of the responses elicited by indi- vidual stimuli and these responses can be enhanced or depressed by resonant and adaptation phenomena. The interplay between these effects seems to depend on the stimulus repetition rate.

\section{Acknowledgements}

This research was supported by CNR and MPI grants. The authors wish to thank Prof. Ermanno Manni for critically reading the manuscript and J.L. Mente for editorial assistance in preparing the English text.

\section{References}

Azzena, G.B., Conti, G., Santarelli, R., Ottaviani, F., Paludetti, G., Maurizi, M. (1995) Generation of the human auditory steady-state responses (SSRs). I. Stimulus rate effects. Hear. Res. 83, 1-8.

Basar, E., Demir, N., Gönder, A. and Ungan, P. (1979a) Combined dynamics of EEG and evoked potentials. I. Studies of simultaneously recorded EEG-EPograms in the auditory pathway, reticular formation and hyppocampus of cat brain during waking stage. Biol. Cybern. 34, 1-19.

Basar, E., Durusan, R., Gönder, A. and Ungan, P. (1979b) Combined dynamics of EEG and evoked potentials. II. Studies of simultaneously recorded EEG-EPograms in the auditory pathway, reticular formation and hyppocampus of cat brain during sleep. Biol. Cybern. 34, 21-30.

Basar, E., Rosen, B., Basar-Eroglu, C. and Greitschus, F. (1987) The associations between $40 \mathrm{~Hz}-\mathrm{EEG}$ and the middle latency response of the auditory evoked potential. Int. J. Neurosci. 33, 103-117.

Bressler, S.L. and Freeman, W.J. (1980) Frequency analysis of olfactory system EEG in cat, rabbit and rat. Electroenceph. Clin. Neurophysiol. 50, 19-24.

Erwin, R.J. and Buchwald, J.S. (1986) Midlatency auditory evoked responses: differential effects of sleep in the human. Electroenceph. Clin. Neurophysiol. 65, 383-392.

Galambos, R., Makeig, S. and Talmachoff, P.J. (1981) A $40-\mathrm{Hz}$ auditory potential recorded in the human scalp. Proc. Natl. Acad. Sci. U.S.A. 78, 2643-2647.

Galambos, R. (1982) Tactile and auditory stimuli presented at high rates $(30-50$ per sec) produce similar event related potentials. Ann. N.Y. Acad. Sci. 88, 722-728.

Hari, R., Hämäläinen, M. and Joutsiniemi, S.L. (1989) Neuromagnetic steady-state responses to auditory stimuli. J. Acoust. Soc. Am. 86, 1033-1039.

ISO 389. (1985) Acoustics standard reference zero for the calibration of pure tone audiometers. International Organization for Standardization, Switzerland.

Kavanagh, K.T.; Harker, L.A. and Tyler, R.S. (1984) Auditory brainstem and middle latency responses. I. Effects of response filtering and waveform identification. Ann. Otol. Rhinol. Laryngol. 93, $1-8$.

Makeig, S. and Galambos, R. (1989) The $40-\mathrm{Hz}$ band evoked response lasts $150 \mathrm{msec}$ and increases in size at slow rates. Soc. Neurosci. Abstr. 15, 113.

Mäkelä, J.P. and Hari, R. (1987) Evidence for cortical origin of the $40 \mathrm{~Hz}$ auditory evoked response in man. Electroenceph. Clin. Neurophysiol. 66, 539-546.

Mäkelä, J.P., Karmos, G., Molnár, M., Csépe, V. and Winkler, I. (1990) Steady-state responses from the cat auditory cortex. Hear. Res. 45, 41-50. 
Mendel, M.I. and Goldstein, R. (1971) Early components of the averaged electroencephalic response to constant level clicks during all-night sleep. J. Speech Hear. Res. 14, 829-840.

Ottaviani, F., Paludetti, G., Grassi, S., Draicchio, F., Santarelli, R., Serafini, G. and Pettorossi, V.E. (1990) Auditory steady-state responses in the rabbit. Audiology 29, 212-218.

Pantev, C., Makeig, S., Hoke, M., Galambos, R., Hampson, S. and Gallen, C. (1991) Human auditory evoked gamma-band magnetic fields. Proc. Natl. Acad. Sci. 88, 8996-9000.

Pantev, C., Elbert, T., Makeig, S., Hampson, S., Eulitz, C. and Hoke, M. (1993) Relationship of transient and steady-state auditory evoked fields. Electroenceph. Clin. Neurophysiol. 88, 389-396.

Picton, T.W.; Hillyard, S.A., Krausz, H.I. and Galambos R. (1974) Human auditory evoked potentials. I. Evaluation of components. Electroenceph. Clin. Neurophysiol. 36, 179-190.

Picton, T.W. and Smith, A.D. (1978) The practice of evoked potential audiometry. Otolaryngol. Clin. North Am. 11, 263-282.
Picton, T.W., Champagne, S.C. and Kellett, A.J.C. (1992) Human auditory evoked potentials recorded using maximum length sequences. Electroenceph. Clin. Neurophysiol. 84, 90-100.

Plourde, G., Stapells, D.R. and Picton, T.W. (1991) The human auditory steady-state evoked potentials. Acta Otolaryngol.(Stockh.) Suppl. 491, 153-160.

Stapells, D.R., Linden, D., Suffield, J.B., Hamel, G. and Picton, T.W. (1984) Human auditory Steady-State potentials. Ear Hear. $5,105-113$.

Stapells, D.R., Galambos, R., Costello, J.A. and Makeig, S. (1988) Inconsistency of auditory middle latency and steady-state responses in infants. Electroenceph. Clin. Neurophysiol. 71, 289295.

Thornton, A.R., Mendel, M.I. and Anderson, C.V. (1977) Effects of stimulus frequency and intensity on the middle components of the averaged auditory electroencephalic response. J. Speech Hear. Res. 20, 81-94. 